\title{
Neural Correlates of the Automatic Processing of Threat Facial Signals
}

\author{
Adam K. Anderson, ${ }^{1}$ Kalina Christoff, ${ }^{1}$ David Panitz, ${ }^{1}$ Eve De Rosa, ${ }^{2}$ and John D. E. Gabrieli ${ }^{1,3}$ \\ Departments of ${ }^{1}$ Psychology, ${ }^{2}$ Psychiatry, and ${ }^{3}$ Neuroscience, Stanford University, Stanford, California 94305
}

The present study examined whether automaticity, defined here as independence from attentional modulation, is a fundamental principle of the neural systems specialized for processing social signals of environmental threat. Attention was focused on either scenes or faces presented in a single overlapping display. Facial expressions were neutral, fearful, or disgusted. Amygdala responses to facial expressions of fear, a signifier of potential physical attack, were not reduced with reduced attention to faces. In contrast, anterior insular responses to facial expressions of disgust, a signifier of potential physical contamination, were reduced with reduced attention. However, reduced attention enhanced the amygdala response to disgust expressions; this enhanced amygdala response to disgust correlated with the magnitude of attentional reduction in the anterior insular response to disgust. These results suggest that automaticity is not fundamental to the processing of all facial signals of threat, but is unique to amygdala processing of fear. Furthermore, amygdala processing of fear was not entirely automatic, coming at the expense of specificity of response. Amygdala processing is thus specific to fear only during attended processing, when cortical processing is undiminished, and more broadly tuned to threat during unattended processing, when cortical processing is diminished.

Key words: amygdala; insula; fear; disgust; attention; emotion; faces; fMRI

\section{Introduction}

Facial expressions serve as important social signals of imminent environmental conditions. It is now known that distinct expressions signaling environmental threat draw on distinct neural substrates specialized for their evaluation. Patient and neuroimaging studies suggest that the amygdala is critical for evaluating fearful facial expressions (Adolphs et al., 1994; Breiter et al., 1996; Morris et al., 1996; Whalen et al., 1998). Similar evidence indicates that the anterior insula, a region of primary gustatory cortex substantially connected with the amygdala (Mesulam and Mufson, 1982), is specialized for evaluating facial expressions of disgust (Phillips et al., 1997, 1998; Calder et al., 2000). The evidence that expressions of fear, a form of threat related to physical attack (Gray, 1987), and expressions of disgust, a form of threat related to physical contamination and disease (Rozin and Fallon, 1987), draw on specialized brain substrates is one measure of the special informational status the human brain places on social signals of potential environmental threats. Another measure of the special status of social signals of threat is the proposal that their processing occurs automatically, proceeding largely independently of attention (Ohman et al., 2001) and awareness (Esteves et al., 1994). Evidence for such automaticity has been shown by how the amygdala responds to fearful faces during diminished attention (Vuilleumier et al., 2001, 2002) and awareness (Whalen et al.,

\footnotetext{
Received Jan. 28, 2003; revised April 15, 2003; accepted April 17, 2003.

This work was supported by National Institute of Mental Health Grant MH12829-01 and by McDonnell-Pew Program in Cognitive Neuroscience Grant 20002024.

Correspondence should be addressed to Dr. Adam K. Anderson, Department of Psychology, 100 St. George Street, University of Toronto, Toronto, Ontario, Canada M5S3G3. E-mail: anderson@psych.utoronto.ca.

Copyright $\odot 2003$ Society for Neuroscience $\quad$ 0270-6474/03/235627-07\$15.00/0
}

1998). Amygdala activation to fearful faces has also been shown in patients with visual neglect (Vuilleumier et al., 2002) and in patients with cortical blindness (Morris et al., 2001).

However, it is unknown whether automaticity is unique to amygdala fear processing or whether it is a fundamental principle of neural systems dedicated to threat signals. There is little, if any, evidence about the attentional properties of the neural processing of disgust, or any facial expression other than fear. Furthermore, recent challenges to the preattentive nature of amygdala processing (Pessoa et al., 2002a,b) suggest that the precise nature of automatic processing in the amygdala is unknown. For instance, it has been proposed that fear responses draw on two distinct pathways to the amygdala: one pathway cortically and another subcortically mediated (LeDoux, 1996; Morris et al., 1999, 2001). By circumventing the cortex, the subcortical pathway may be more rapid and automatic, but should be at the expense of a more detailed cortical analysis of the stimulus (Jarrell et al., 1987; LeDoux, 1995). Thus, amygdala automatic processing may be qualitatively distinct from processing under conditions of full awareness, occurring at the expense of its specificity for fear.

To address these issues, the present study used event-related functional magnetic resonance imaging (fMRI) to examine how attention influences amygdala and anterior insular processing of fear and disgust. Manipulations of visual attention result in a pronounced modulation of extrastriate responses (Corbetta et al., 1990; Haxby et al., 1994; Wojciulik et al., 1998; O'Craven et al., 1999). If automaticity, defined here as the lack of reduction in activation with reduced attention, is a fundamental principle of the neural processing of social signals of environmental threat, then lack of attentional modulation should extend to both amygdala processing of fear and anterior insular processing of disgust. 
Furthermore, if automatic processing is qualitatively similar to processing taking place during full attention, then reduced attention should not influence the response specificity in the amygdala and/or anterior insula.

\section{Materials and Methods}

Participants. Informed consent to take part in a study approved by the Stanford University Panel on Human Subjects in Medical Research was obtained from each subject (three men, nine women; mean age, 22.1 years; range, 18-29).

Stimuli. Stimuli consisted of photographs either of fearful, disgusted, or neutral faces superimposed on pictures of places (see Fig. 1a). For the purposes of decreasing stimulus repetition, which is thought to relate to pronounced amygdala habituation (Breiter et al., 1996), increasing the number of unique facial exemplars was emphasized. Facial expression stimuli were taken from the Facial Affect Series and supplemented by additional appropriately normed exemplars, resulting in three facial expression types for 18 distinct individuals ( 9 male, 9 female). Place stimuli consisted of photos of 18 interiors and 18 exteriors of buildings. Superimposition was achieved by rendering each of the faces and places semitransparent. All stimuli were standardized for luminosity, contrast, and transparency. All background place stimuli were $300 \times 300$ pixels in size (at $72 \mathrm{dpi}$ ) with faces presented in an oval aperture $\sim 200 \times 250$ in size, which occluded gender stereotypic features such as hair and facial shape. Stimuli were created such that face gender, expression, and underlying place (interior and exterior) were completely crossed, yielding 108 independent stimuli. Across the course of scanning, each of these stimuli were presented once during attended and once during unattended conditions for a total of 216 trials. Stimuli were presented using a magnetcompatible back-projector (Resonance Technology, Van Nuys, CA).

Task design. We used an intermixed trial event-related design. On each trial, participants were first presented with central fixation $(1 \mathrm{sec})$, which was replaced by a color-coded prompt $(750 \mathrm{msec})$ that indicated whether to make a male/female judgment (attend to the face) or an inside/outside judgment (attend to the place) of a subsequently presented stimulus. After $250 \mathrm{msec}$, the superimposed face/place stimulus was presented for $750 \mathrm{msec}$. Participants were asked to indicate, as quickly and as accurately as possible, either the gender of the face (attend condition) or to indicate whether the place was the inside or outside of a building (unattend condition). We opted to use such an object attentional selection task to limit the role of eye movements, which would be a larger concern in spatialselection tasks. To ensure appropriate averaging of the overlapping hemodynamic responses from distinct trial types, trials were presented in a fixed randomized order that counterbalanced trial type history.

After the test scans, participants took part in a fusiform face area (FFA) and parahippocampal place area (PPA) localizer scan. On each trial, subjects were first presented with central fixation $(1 \mathrm{sec})$ and then an image of a neutral face ( 12 male, 12 female) or a building ( $1 \mathrm{sec}$ ). Images were presented in a fixed random intermixed order and participants were asked to simply indicate whether a picture of a face or a place was presented. All stimuli were distinct from those used in the previous test scans.

Image acquisition and analysis. Participants were scanned with a 3 tesla Signa (General Electric, Milwaukee, WI) scanner with a prototype head coil. Foam padding placed around the head was used to minimize movement. Every second we acquired seventeen $4 \mathrm{~mm}$ slices ranging from the body of the corpus callosum to the ventral surface of the anterior temporal lobe using a $\mathrm{T}_{2}{ }^{*}$-weighted spiral pulse sequence (in-plane resolution, $3.755 \mathrm{~mm}$; repetition time, $1000 \mathrm{msec}$; echo time, $30 \mathrm{msec} ; 60^{\circ}$ flip angle, $24 \mathrm{~cm}$ field of view; $64 \times 64$ matrix acquisition). The intertrial interval (ITI) was $8 \mathrm{sec}$. Four separate scans collected 1728 frames (288 per condition), with 36 repetitions for each of the six trial types. Two dummy trials were added at the beginning of each session to avoid scanner equilibration effects. The same slice prescription and scanning parameters were used in the subsequent localizer scan, with the exception of an increased ITI of $20 \mathrm{sec}$. One session collected 960 frames (480 place, 480 face), resulting in 24 repetitions of each trial type. $\mathrm{T}_{1}$-weighted spin echo images were acquired for all slices that received functional scans as well as an additional $\mathrm{T}_{1}$-weighted whole-brain anatomy for the purposes of normalization of functional data into common stereotactic space.

Statistical analysis was performed using statistical parametric mapping software (SPM99; Wellcome Department of Cognitive Neurology, London, UK). After image reconstruction, motion estimation, realignment, slice-time correction, normalization, and spatial smoothing (full width at half-maximum, $6 \mathrm{~mm}$ ) were performed. During normalization, voxels were resampled to $2 \times 2 \times 4 \mathrm{~mm}$. The presentation of each face/place stimulus was modeled by a canonical hemodynamic response function (hrf). For each individual, contrast images were calculated by applying appropriate weights to the parameter estimates for the regressor of each event type. Group analysis for identification of the amygdala and anterior insular regions of interest (ROIs) was performed on these contrast images, which were submitted to a one-sample $t$ test across the 12 subjects, with subjects entered as a random effect. Group contrast images were overlaid onto the SPM99 high-resolution $T_{1}$ individual template image for viewing. Coordinates of activation were converted from Montreal Neurological Institute to Talairach space.

ROI delineation. The first phase of analysis was to replicate four separate findings for purposes of ROI delineation: (1) amygdala activation to fear faces, (2) anterior insular activation to disgust faces, (3) FFA activation to faces, and (4) PPA activation to places. Accordingly, the ROIs were localized with relatively liberal uncorrected criterion $(p<0.01)$; subsequently, signals from these regions were submitted for examination of the main hypotheses. The amygdala ROI was defined by the contrast of fear relative to neutral faces when subjects were attending to faces (cluster extent threshold, 10 voxels). The anterior insular ROI was defined by disgust relative to neutral faces when subjects were attending to faces (extent threshold, 10 voxels). Post-test localizer data were used to identify the FFA and PPA for each subject (extent threshold, 5 voxels). Each subject's FFA and PPA were defined by a combination of functional and structural features. Right-hemisphere voxels confined to the middle fusiform gyrus that were more active while viewing faces compared with places were considered to be the FFA. In addition, voxels lateral to the occipital temporal sulcus and confined to the inferior and middle temporal gyri that were more active while viewing faces compared with places were considered to be face-responsive regions within the lateral occipital complex, referred to here as the LOCf. Bilateral voxels confined to the parahippocampal gyrus that were more active while viewing places compared with faces were considered to be the PPA.

ROI signal analyses. For each subject, signal change indexed by the fit of canonical hrf was extracted for each of the eight data frames for each of the six trial types averaged across 36 trial repetitions and then submitted to statistical analysis. The $\alpha$ value for analysis of ROI signal was set at $p<0.01$.

\section{Results}

\section{Behavioral performance}

Observers were less accurate in making gender judgments on faces than location judgments on places $(87.3 \pm 1.1$ vs $79.3 \pm$ $\left.1.4 \% ; F_{(1,11)}=14.07 ; p<0.003\right)$. Gender judgments were influenced by emotional expression (neutral, $75.7 \pm 1.7 \%$; disgust, $81.9 \pm 2.7 \%$; fear, $\left.80.3 \pm 2.3 \% ; F_{(2,22)}=3.62 ; p<0.05\right)$. Accuracy in making place judgments was not influenced by the stimulus content of the to-be-ignored faces (neutral, $87.5 \pm 2.2 \%$; disgust, $86.6 \pm 1.6 \%$; fear, $\left.87.7 \pm 2.2 \% ; F_{(2,22)}=0.24 ; p>0.79\right)$. Analysis of response latency revealed no significant difference in the times taken to make face and place judgments $(820 \pm 49$ vs $754 \pm 46$ msec; $\left.F_{(1,11)}=2.58 ; p>0.14\right)$. The response latency for gender judgments was influenced by emotional expression (neutral, $786 \pm 81 \mathrm{msec}$; disgust, $755 \pm 69 \mathrm{msec}$; fear, $811 \pm 89 \mathrm{msec}$; $\left.F_{(2,22)}=4.46 ; p<0.03\right)$. The response latency for making place judgments was not influenced by the stimulus content of the to-be-ignored faces (neutral, $732 \pm 81 \mathrm{msec}$; disgust, $727 \pm 77$ msec; fear, $\left.753 \pm 86 ; F_{(2,22)}=1.60 ; p>0.23\right)$.

Although facial expression did influence gender judgment accuracy and latency, and may have contributed to the magnitude 

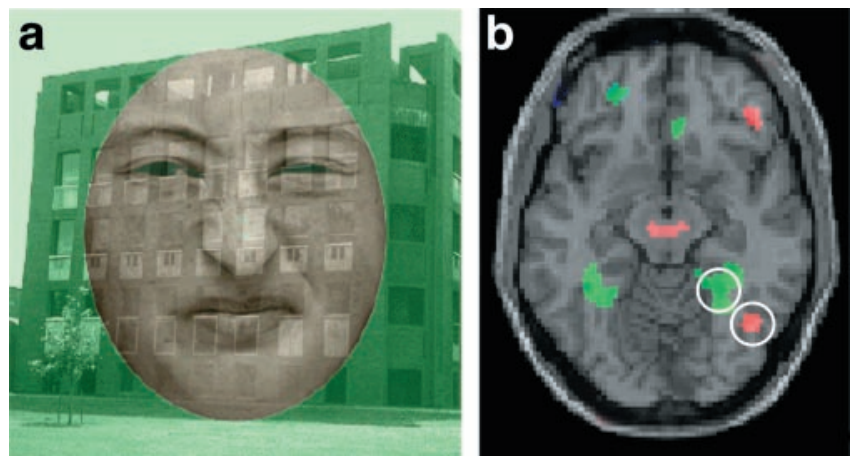

Figure 1. Face-place object selection attention task. $a$, Example stimulus. Observers were presented with color-coded superimposed faces (disgusted, fearful, and neutral expressions in red) and places (inside and outside of buildings in green). Before each test stimulus, observers were presented with a color-coded prompt indicating which task they were to perform on that trial: indicate the gender of the face (attend trials) or indicate the location of the place (unattend trials). $b, A$ representative subject demonstrated a greater response when attending to places (in green) in a bilateral region along the collateral sulcus, consistent with the PPA and a greater response when attending to faces (in red) in the right middle fusiform gyrus, consistent with the FFA.

of blood-oxygen level-dependent (BOLD) response when faces were attended, critically, performance on place judgments (when subjects were instructed to ignore faces) did not differ between face types. This suggests that attention was equally divided for unattended neutral, disgust, and fear face trials, so that performance differences did not account for differences in BOLD responses on unattended trials.

\section{Effect of attention on extrastriate responses}

Confirming previous results, decreased attention resulted in a substantial reduction in cortical activations to both faces and places. A region functionally defined as more responsive to faces than places in the right middle fusiform gyrus, consistent with the FFA, demonstrated a greater response (average, $4-7 \mathrm{sec}$ from stimulus onset) when subjects were attending to faces and not places $\left(F_{(1,154)}=386.69 ; p<0.0001\right)$ (Fig. $\left.1 b\right)$. Conversely, a bilateral region functionally defined as more responsive to places than faces along the collateral sulcus, consistent with the PPA, demonstrated a greater response when subjects were attending to places and not to faces $\left(F_{(1,154)}=74.76 ; p<0.0001\right)$ (Fig. 1b).

\section{Effect of attention on amygdala response}

When subjects were attending to faces, a comparison of fear relative to neutral faces resulted in a discrete activation in the right amygdala (43 voxels, at a peak height $x, 22 ; y, 1 ; z,-28$; in Talairach coordinates, $\left.F_{(1,11)}=20.52 ; p<0.0001\right)$ (Fig. $2 a$ ). During attended conditions, the peak response in this functionally defined amygdala ROI was greater for fearful expressions than either disgusted (fear vs disgust, $F_{(1,154)}=27.93 ; p<0.0001$ ) or neutral expressions (fear vs neutral, $F_{(1,154)}=40.22 ; p<0.0001$ ); disgusted and neutral expressions did not differ $\left(F_{(1,154)}=1.12\right.$; $p>0.28$ ) (Fig. 2b). Thus, the amygdala response was specific to fear and did not generalize to disgust. The magnitude of the amygdala response to fearful faces was not significantly modulated by attention $\left(F_{(1,154)}=0.24 ; p>0.62\right)$, remaining greater than neutral expressions (fear vs neutral, $F_{(1,154)}=25.58$; $p<$ $0.0001)$ during inattention.

However, the amygdala demonstrated a surprising increase in response to expressions of disgust during unattended relative to attended conditions $\left(F_{(1,154)}=48.67 ; p<0.0001\right)$ (Fig. 3). Be-
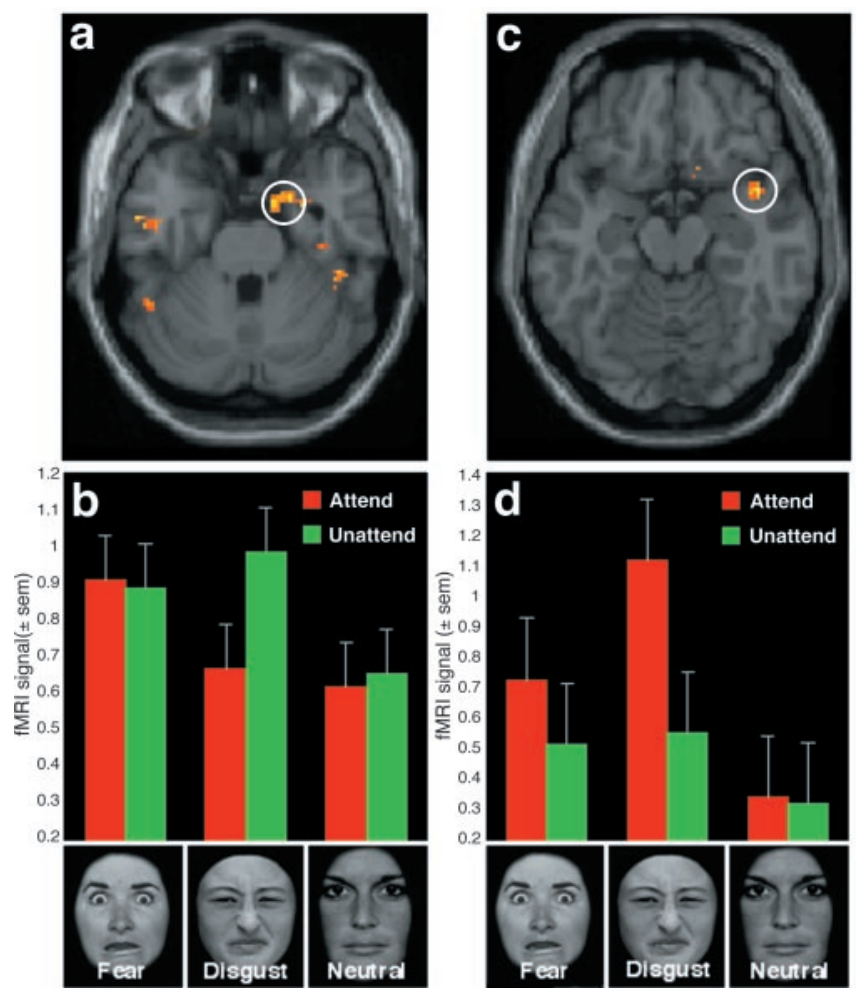

Figure 2. Attentional dependence of amygdala and anterior insular responses to facial expressions. $a$, The amygdala was functionally defined by the group level contrast of fear relative to neutral trials when faces were attended. This resulted in a prominent activation in the right amygdala (at a peak height $\left.x, 22 ; y, 1 ; z,-28 ; F_{(1,11)}=20.52 ; p<0.0001\right) . b$, Effect of stimulus and attention on amygdala response. Peak amygdala response is displayed for each facial stimulus type during attended (red) and unattended (green) conditions. Attention did not significantly reduce the magnitude of amygdala response to fear, but the enhanced response to disgust during reduced attention suggests attention influenced the specificity of amygdala response. $c$, The insula was functionally defined by contrasting activation on disgust trials compared with neutral trials when faces were attended. This resulted in a prominent activation in the right anterior insula (at a peak height $x, 44 ; y, 5 ; z,-14 ; F_{(1,11)}=32.72, p<0.0001$ ). $d$, Effect of stimulus and attention on anterior insular response. Peak anterior insular response is displayed for each facial stimulus type during attended (red) and unattended (green) conditions. Reduced attention significantly reduced the magnitude of anterior insular response to disgust.

cause of this increased response to disgust, when faces were unattended, the amygdala response magnitude to fear was no longer significantly greater than that to disgust, with a tendency for a greater response to disgust $\left(F_{(1,154)}=4.75 ; p>0.031\right)$. Both fear $\left(F_{(1,154)}=31.89 ; p<0.0001\right)$ and disgust $\left(F_{(1,154)}=54.99 ; p<\right.$ $0.0001)$ resulted in greater responses relative to neutral expressions (Fig. $2 b$ ). Thus, inattention did not significantly reduce the amygdala response to fear faces, but did significantly enhance the amygdala response to disgust faces.

\section{Effect of attention on anterior insular response}

When subjects were attending to faces, a comparison of disgust relative to neutral faces resulted in activation in the right anterior insula (22 voxels, at peak height $x, 44 ; y, 6 ; z,-16 ; F_{(1,11)}=32.72$; $p<0.0001$ ) (Fig. 2c). During attended conditions, the peak response in this insular ROI was greater for disgusted expressions than for neutral expressions (disgust vs neutral, $F_{(1,154)}=117.13$; $p<0.0001)$. Fear responses were also greater than neutral in this region (fear vs neutral, $F_{(1,154)}=29.04 ; p<0.0001$ ), but there remained a greater response to disgusted than to fearful faces $\left(F_{(1,154)}=29.53 ; p<0.0001\right)$. The magnitude of insular response 

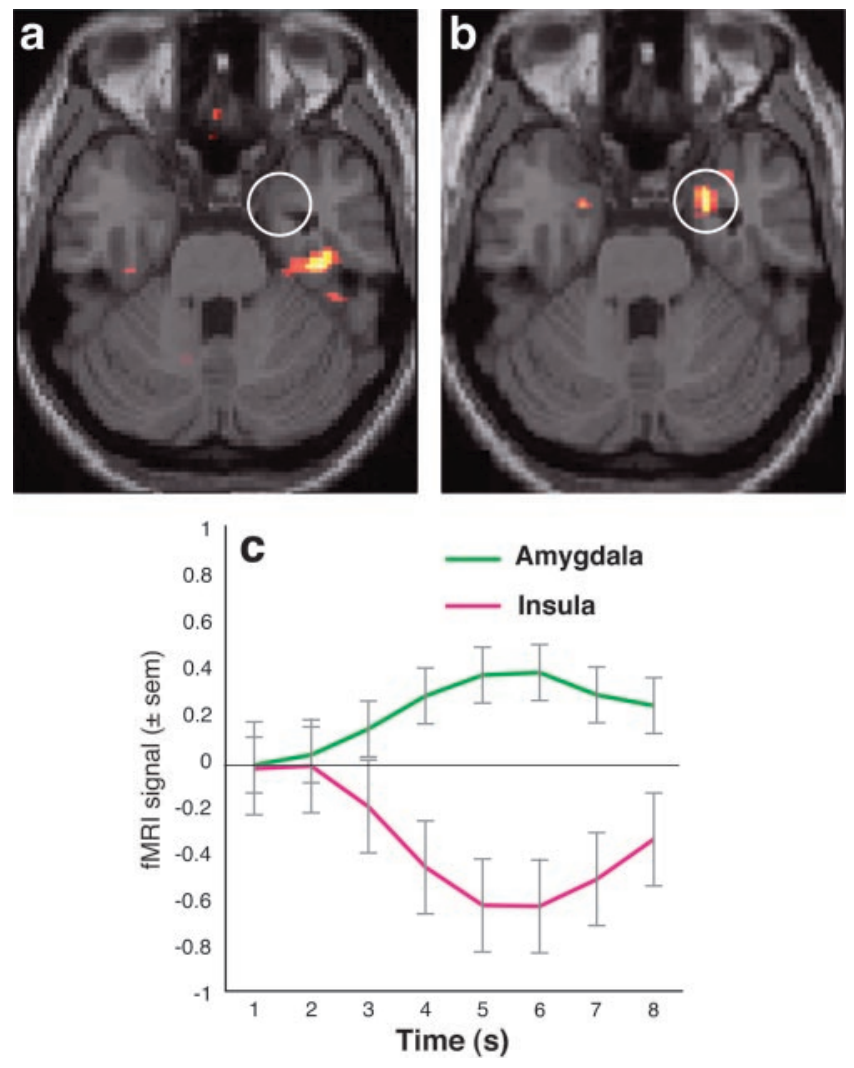

Figure 3. Response to disgust faces when unattended. $a$, Amygdala response to disgust relative to neutral faces when observers were attending to faces. No significant activation was found when faces were attended. $b$, Amygdala response to disgust relative to neutral faces when observers were attending to places. Activation was present when disgust faces were unattended. c, Time course of the disgust response difference score (unattended minus attended). A negative deflection of time course represents a decreased response when faces were attended. A positive deflection represents an increased response when faces were unattended. An inverse effect of attention on anterior insula and amygdala response to disgust faces peaked $\sim 6$ sec after the stimulus onset.

to disgust $\left(F_{(1,154)}=61.88 ; p<0.0001\right)$ and fear $\left(F_{(1,154)}=8.64\right.$; $p<0.004)$ were both significantly reduced during unattended compared with attended conditions. During inattention, the magnitude of insular response was no longer greater to disgust than to fear $\left(F_{(1,154)}=0.26 ; p>0.6\right)$, but did remain greater to disgust than neutral $\left(F_{(1,154)}=10.58 ; p<0.002\right)$ (Fig. $\left.2 d\right)$. These results indicate that both the magnitude and the specificity of the insular response to disgust were significantly reduced with diminished attention.

In addition to the insula, patient and neuroimaging studies suggest a role of the striatum in evaluating disgust expressions (Sprengelmeyer et al., 1996; Phillips et al., 1997, 1998). When we reduced our statistical and extent thresholds $(p<0.05$ and 5 voxels), activation in a contiguous bilateral ventral striatal region was greater for disgust than for neutral expressions during attended conditions (peak height on the right at $x, 16 ; y, 18 ; z,-12$; $F_{(1,11)}=25.91 ; p<0.0001$; peak height on the left at $x,-2 ; y, 10$; $\left.z,-8 ; F_{(1,11)}=32.15 ; p<0.0001\right)$. Like the anterior insular response, inattention resulted in a substantially reduced striatal response to disgust $\left(F_{(1,154)}=13.41 ; p<0.0003\right)$.

\section{Effect of attention on cortico-amygdala interactions}

The inverse relationship between cortical response to disgust (diminished with diminished attention) and the amygdala response to disgust (enhanced with diminished attention) is consistent with the notion that cortical processing can influence the breadth/narrowness of the amygdala response tuning. To examine this hypothesis more closely, we assayed the relation between the amygdala response and how attention influences cortical responsiveness to facial signals of threat in three functionally defined face responsive regions: (1) within the FFA, a region specialized for face processing; (2) within the LOCf, an area lateral to the FFA, purportedly within the lateral occipital complex (GrillSpector et al., 2001), a region specialized for shape processing; and (3) the anterior insula, a region specialized for disgust face processing.

During attended conditions, responses were greater for fearful relative to neutral faces in the FFA $\left(F_{(1,154)}=82.94 ; p<0.0001\right)$ and $\operatorname{LOCf}\left(F_{(1,154)}=48.37 ; p<0.0001\right)$. These greater responses to fear were significantly diminished under unattended relative to attended conditions in the FFA $\left(F_{(1,154)}=168.17 ; p<0.0001\right)$ and LOCf $\left(F_{(1,154)}=61.47 ; p<0.0001\right)$, with response magnitudes in the FFA $\left(F_{(1,154)}=3.56 ; p>0.06\right)$ and $\operatorname{LOCf}\left(F_{(1,154)}=\right.$ $1.67 ; p>0.19)$ no longer greater for fearful versus neutral expressions. During attended conditions, responses were also greater for disgusted relative to neutral faces in the FFA $\left(F_{(1,154)}=32.96\right.$; $p<0.0001)$ and LOCf $\left(F_{(1,154)}=32.96 ; p<0.0001\right)$. These greater responses to disgust were also diminished during unattended relative to attended conditions in the FFA $\left(F_{(1,154)}=\right.$ 82.94; $p<0.0001)$ and $\operatorname{LOCf}\left(F_{(1,154)}=182.00 ; p<0.0001\right)$, with FFA and LOCf responses to disgust being numerically smaller than that of neutral expressions during unattended conditions. Thus, like the anterior insula, FFA, and LOCf responses to fear and disgust were significantly reduced with diminished attention (Fig. 4).

This inverse effect of attention on cortical (FFA, LOCf, and anterior insula) and amygdala responses to disgust suggests that the loss of fear specificity in the amygdala is related to diminished cortical processing of disgust during inattention. To examine such putative cortico-amygdala interactions, we assessed individual differences in the magnitude of attentional modulation (attended vs unattended) of the amygdala response to disgust and its correlation with magnitude of attentional modulation of FFA, LOCf, and the anterior insular responses to disgust. Although all three cortical regions demonstrated substantial attentional modulation of disgust responses, multiple regression analysis revealed that only the anterior insula (standardized $\beta$ coefficient $=$ $\left.-0.471 ; F_{(1,93)}=19.58 ; p<0.0001\right)$, and neither FFA $(\beta=$ $\left.-0.17 ; F_{(1,93)}=2.22 ; p>0.13\right)$ nor LOCf $\left(\beta=0.08 ; F_{(1,93)}=\right.$ $0.41 ; p>0.52)$, was significantly negatively associated with enhanced amygdala response to disgust. That is, subjects who demonstrated the largest attention-related decrease in anterior insular response tended to be the same as those who showed the largest increase in the amygdala response to disgust. This association between attentional modulation of amygdala and insular responses was stimulus dependent. Consistent with the attention-independent amygdala response to fear, amygdala responses were not significantly correlated with attentiondependent anterior insular responses to fear $\left(r=-0.10 ; F_{(1,95)}=\right.$ $0.95 ; p>0.33)$.

Responses to disgust in the FFA, LOCf, and anterior insula all demonstrated a pronounced reduction in response with reduced attention. In addition, this association suggests that anterior insular responses to disgust may be critically dependent on extrastriate face processing. To examine this possibility further, we assayed the relation between individual differences in the magnitude of attentional modulation of FFA, LOCf, and anterior insular responses to disgust. A multiple regression analysis revealed 

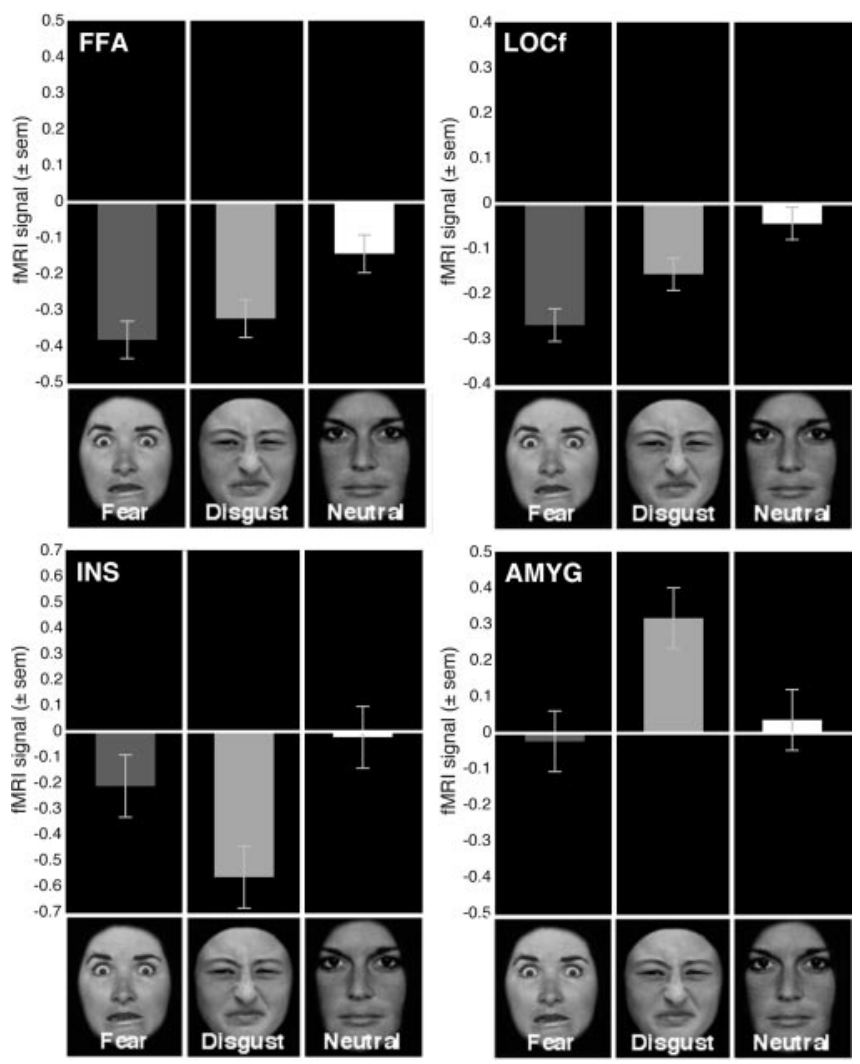

Figure 4. Effect of inattention on FFA, LOCf, anterior insula (INS), and amygdala (AMYG) ROI responses to facial expressions. Bars represent the difference score between attended and unattended conditions (unattend minus attend) for each fear, disgust, and neutral face. The predominant effect of inattention was to reduce cortical responsiveness in the FFA, LOCf, and insula. In contrast, the amygdala demonstrated a marked increased response to disgust.

that the $\operatorname{LOCf}\left(\beta=0.53 ; F_{(1,93)}=26.23 ; p<0.0001\right)$, but not the FFA $\left(\beta=-0.17 ; F_{(1,93)}=2.34 ; p>0.12\right)$, was significantly positively associated with a reduced anterior insular response to disgust.

\section{Discussion}

Consistent with the notion that the amygdala processes fear automatically, the magnitude of the amygdala response to facial signals of fear was not significantly reduced with reduced attention, despite reduced responses to fear in multiple cortical regions. However, such automaticity did not extend to all forms of facial threat processing: the magnitude of anterior insular response to facial signals of disgust was substantially reduced with reduced attention. That automatic processing did not extend to both amygdala processing of fear and anterior insula processing of disgust demonstrates that automaticity is not a fundamental principle of neural systems dedicated to the processing of facial expressions more generally, and facial expressions related to threat in particular. Automaticity appears unique to amygdala processing of social signals of fear. However, amygdala attentional independence may not be complete. The present study found that amygdala processing of fear was not entirely automatic, coming at the expense of specificity of response. During inattention, the amygdala demonstrated a markedly enhanced response to disgust. This finding suggests there are important limitations on what precise affective features the amygdala encodes automatically. Thus, amygdala automatic processing is not specific to fearful faces, but rather, may be confined to more coarse affective properties of faces, such as their valence or arousal/intensity.

In contrast to the present and previous studies, Pessoa et al. (2002a) have shown abolished cortical and amygdala responses to fear faces under conditions of extreme attentional load. Such contradictory results can be reconciled if we consider that different levels of attentional load will result in the modulation of activity at different levels of the nervous system. Indeed, given similar attentional load, there are more pronounced modulations in later visual cortical processing stages [e.g., middle temporal (MT)] relative to earlier stages (e.g., V1) (Kastner et al., 1998, 2001). Severe attentional depletion may then result in modulations very early in processing, before cortical processing (O'Connor et al., 2002) such as in the thalamic relays to the amygdala, functionally cutting off the sensory inputs of the amygdala. This would be consistent with demonstrations of preserved amygdala fear responses in patients with striate cortex lesions (Morris et al., 2001). In the context of the present results, the automaticity of amygdala processing of fear is not all-ornone, but a matter of degree. Relative to PPA processing of places, FFA processing of faces, and anterior insula processing of disgust, the magnitude of the amygdala response to fear demonstrates substantial attentional independence.

The pronounced reduction in extrastriate response during inattention contrasted with the amygdala maintenance of response to fear. This is consistent with fear processing in the amygdala occurring independently of extrastriate face processing (Morris et al., 2001; Vuilleumier et al., 2001). Although the magnitude of the amygdala response to fear takes place independently of extrastriate face processing, the specificity of the amygdala response to fear may remain critically dependent on cortical processing. The enhanced amygdala response to expressions of disgust during decreased attention is exceptional with respect to an extensive body of evidence showing reductions of brain response with reduced attention (Corbetta et al., 1990; Haxby et al., 1994; Wojciulik et al., 1998; O'Craven et al., 1999). This enhanced response to disgust may be a reflection of diminished cortical influences on the amygdala. Although previous studies have suggested that there are significant amygdala modulatory influences on cortical perceptual processing (Anderson and Phelps, 2001; Morris et al., 1998a, 1998b), the present findings suggest that cortical processing can significantly modulate the amygdala response (Phelps et al., 2001; Ochsner et al., 2002).

To our knowledge, this interaction between the automaticity and the specificity of the amygdala response provides the first human evidence supporting an important proposed functional consequence of having two parallel pathways to the amygdala: one subcortically mediated and one cortically mediated (LeDoux, 1996). As proposed by LeDoux (1996), by circumventing the cortex, a shorter thalamo-amygdala pathway processes information in a more rapid and automatic manner. By engaging the cortex, a longer thalamo-cortico-amygdala pathway allows for more detailed processing of the stimulus, but in a less rapid, and as we propose here, in an attention-limited manner. An important consequence of bypassing cortical processing is that the automaticity of the subcortical pathway should hypothetically come with a cost, at the expense of more fine-grained cortical analysis (Thompson, 1962). Indeed, studies in monkeys have shown altered amygdala discrimination of visual stimuli after reversible cooling of the inferotemporal cortex (Fukuda et al., 1987). Rabbits with lesions of the auditory cortex have demonstrated impaired stimulus discrimination during auditory fear conditioning (Jarrell et al., 1987). In addition to reduced cortical pro- 
cessing, decreased attention is associated with decreased stimulus discriminability (Yeshurun and Carrasco, 1998). Accordingly, reduced cortical responses during inattention can be interpreted as reflecting diminished cortical stimulus analysis that may ultimately limit the ability for the amygdala to resolve stimuli of specific types.

Individual differences in the degree of attentional degradation of processing in the anterior insular cortex were particularly tied to the amygdala loss of fear specificity, being directly related to the magnitude of the enhanced amygdala response to disgust. With its substantial projections to the amygdala (Mesulam and Mufson, 1982), the anterior insula has been thought to convey cortical processing of affective stimulus content to the amygdala (Shi and Davis, 1999; Phelps et al., 2001). The anterior insular cortex may provide the amygdala with more detailed information regarding stimulus affective properties when attention is focused on the stimulus source. The result of diminished affective cortical processing is that the amygdala may respond more liberally to potentially significant stimulus events. In signal detection terminology, with diminished cortical inputs the amygdala may weigh more heavily "hits" and "misses" than "correct rejections" and "false alarms." That is, recognizing (hits) or failing to recognize (misses) an environmental threat (e.g., responding, or not, to a dangerous snake) should be more critical for amygdala processing than recognizing (correct rejection) or failing to recognize (false alarms) an event as not threatening (e.g., responding, or not, to snake-like objects, such as a curvy stick). This bias toward potentially important events is not a reflection of a loss of sensitivity to discriminate between potentially significant and neutral events. During inattention, amygdala activation discriminated both fear and disgust from neutral expressions. Thus, in healthy individuals the amygdala does not "cry wolf" to all stimuli, losing its predictive usefulness. Rather, under conditions of reduced stimulus analysis, the amygdala appears to extend its response to a broader range of potential threats, ensuring that potentially significant events will not be overlooked.

However, this adaptive form of automaticity may not hold in clinical populations in which there is substantial behavioral evidence of overgeneralization of automatic processing to normatively more neutral events (Williams et al., 1996). This overgeneralization in clinical populations has been shown with respect to amygdala processing as well. For instance, relative to nonsocial phobic individuals, patients with social phobia demonstrate more pronounced amygdala response to neutral faces (Birbaumer et al., 1998). Broadening of the amygdala response to other facial expressions beyond fear has also been shown in patients with major depression, with this overgeneralization found to be reversible with treatment (Sheline et al., 2001). In the context of the present results, the broadening of amygdala responsiveness in clinical populations, and its reversibility, may reflect altered cortical modulatory influences on the amygdala response. Evidence of gender differences in the amygdala response also underscores the variable nature of amygdala processing (Cahill et al., 2001; Canli et al., 2002). The subjects in the present study were mostly women, so future studies with larger and gender-balanced samples will be needed to examine whether the present findings apply equally to mean and women.

Why is automaticity unique to the amygdala processing of social signals of fear and does it not extend to other brain regions specialized for social signals of threat? Facial signals of fear, as well as disgust, may serve as important cues for searching one's environment for the source of a potential threat (Whalen, 1998). Fear expressions signal impending attack (e.g., response to a danger- ous animal) (Gray, 1987). Disgust expressions signal potential contamination or poisoning (e.g., rejection of harmful food) (Rozin and Fallon, 1987). The character of the stimulus and response for attack-related threat (stimulus: moving target; response: immediate freezing or flight) versus contaminationrelated threat (stimulus: stationary target; response: further inspection and passive avoidance) differ significantly (Sawchuk et al., 2002). Consistent with this division, predatory animals tend to evoke fear, whereas disgust is associated with animals that do not present significant harm (e.g., spiders, slugs) (Ware et al., 1994). Similarly, fear and disgust demonstrate distinct physiological signatures and action tendencies (Ekman, 1992; Levenson, 1992), with fear associated with increased sympathetic activity (Ekman et al., 1983) and disgust more with parasympathetic activity (Rozin and Fallon, 1987; Levenson, 1992). Commensurate with the prerequisite rapidity of attack-related threat evaluations, the analysis of fear content from faces may occur early on, with relative independence from higher-order attention-limited processes. In contrast, disgust content from faces may have the luxury of occurring later, being dependent on more elaborative and attention-demanding processes. Thus, although selective pressures have promoted the development of specialized neural systems for the processing of social signals of both fear and disgust, selection for automaticity may extend only to fear.

\section{References}

Adolphs R, Tranel D, Damasio H, Damasio A (1994) Impaired recognition of emotion in facial expressions following bilateral damage to the human amygdala. Nature 372:669-672.

Anderson AK, Phelps EA (2001) Lesions of the human amygdala impair enhanced perception of emotionally salient events. Nature 411:305-309.

Birbaumer N, Grodd W, Diedrich O, Klose U, Erb M, Lotze M, Schneider F, Weiss U, Flor H (1998) fMRI reveals amygdala activation to human faces in social phobics. NeuroReport 9:1223-1226.

Breiter HC, Etcoff NL, Whalen PJ, Kennedy WA, Rauch SL, Buckner RL, Strauss MM, Hyman SE, Rosen BR (1996) Response and habituation of the human amygdala during visual processing of facial expression. Neuron 17:875-887.

Cahill L, Haier RJ, White NS, Fallon J, Kilpatrick L, Lawrence C, Potkin SG, Alkire MT (2001) Sex-related difference in amygdala activity during emotionally influenced memory storage. Neurobiol Learn Mem 75:1-9.

Calder AJ, Keane J, Manes F, Antoun N, Young AW (2000) Impaired recognition and experience of disgust following brain injury. Nat Neurosci 3:1077-1078.

Canli T, Desmond JE, Zhao Z, Gabrieli JD (2002) Sex differences in the neural basis of emotional memories. Proc Natl Acad Sci USA 99:10789-10794.

Corbetta M, Miezin FM, Dobmeyer S, Shulman GL, Petersen SE (1990) Attentional modulation of neural processing of shape, color, and velocity in humans. Science 248:1556-1559.

Ekman P (1992) An argument for basic emotions. Cognition Emotion 6:169-200.

Ekman P, Levenson RW, Friesen WV (1983) Autonomic nervous system activity distinguishes among emotions. Science 221:1208-1210.

Esteves F, Dimberg U, Ohman A (1994) Automatically elicited fear: conditioned skin conductance responses to masked facial expressions. Cognition Emotion 8:393-413.

Fukuda M, Ono T, Nakamura K (1987) Functional relations among inferotemporal cortex, amygdala, and lateral hypothalamus in monkey operant feeding behavior. J Neurophysiol 57:1060-1077.

Gray JA (1987) The psychology of fear and stress, Ed 2. New York: Cambridge UP.

Grill-Spector K, Kourtzi Z, Kanwisher N (2001) The lateral occipital complex and its role in object recognition. Vision Res 41:1409-1422.

Haxby JV, Horwitz B, Ungerleider LG, Maisog JM, Pietrini P, Grady CL (1994) The functional organization of human extrastriate cortex: a PETrCBF study of selective attention to faces and locations. J Neurosci 14:6336-6353.

Jarrell TW, Gentile CG, Romanski LM, McCabe PM, Schneiderman N 
(1987) Involvement of cortical and thalamic auditory regions in retention of differential bradycardiac conditioning to acoustic conditioned stimuli in rabbits. Brain Res 412:285-294.

Kastner S, De Weerd P, Desimone R, Ungerleider LG (1998) Mechanisms of directed attention in the human extrastriate cortex as revealed by functional MRI. Science 282:108-111.

Kastner S, De Weerd P, Pinsk MA, Elizondo MI, Desimone R, Ungerleider LG (2001) Modulation of sensory suppression: implications for receptive field sizes in the human visual cortex. J Neurophysiol 86:1398-1411.

LeDoux JE (1995) Emotion: clues from the brain. Annu Rev Psychol 46:209-235.

LeDoux JE (1996) The emotional brain: the mysterious underpinnings of emotional life. New York: Simon \& Schuster.

Levenson RW (1992) Autonomic nervous system differences among emotions. Psychol Sci 3:23-27.

Mesulam MM, Mufson EJ (1982) Insula of the old world monkey. III. Efferent cortical output and comments on function. J Comp Neurol 212:38-52.

Morris JS, Frith CD, Perrett DI, Rowland D, Young AW, Calder AJ, Dolan RJ (1996) A differential neural response in the human amygdala to fearful and happy facial expressions. Nature 383:812-815.

Morris JS, Friston KJ, Buchel C, Frith CD, Young AW, Calder AJ, Dolan RJ (1998a) A neuromodulatory role for the human amygdala in processing emotional facial expressions. Brain 121:47-57.

Morris JS, Friston KJ, Dolan RJ (1998b) Experience-dependent modulation of tonotopic neural responses in human auditory cortex. Proc R Soc Lond B Biol Sci 265:649-657.

Morris JS, Ohman A, Dolan RJ (1999) A subcortical pathway to the right amygdala mediating "unseen" fear. Proc Natl Acad Sci USA 96:1680-1685.

Morris JS, DeGelder B, Weiskrantz L, Dolan RJ (2001) Differential extrageniculostriate and amygdala responses to presentation of emotional faces in a cortically blind field. Brain 124:1241-1252.

O'Connor DH, Fukui MM, Pinsk MA, Kastner S (2002) Attention modulates responses in the human lateral geniculate nucleus. Nat Neurosci 5:1203-1209.

O’Craven KM, Downing PE, Kanwisher N (1999) fMRI evidence for objects as the units of attentional selection. Nature 401:584-587.

Ochsner KN, Bunge SA, Gross JJ, Gabrieli JD (2002) Rethinking feelings: an fMRI study of the cognitive regulation of emotion. J Cogn Neurosci 14:1215-1219.

Ohman A, Lundqvist D, Esteves F (2001) The face in the crowd revisited: a threat advantage with schematic stimuli. J Pers Soc Psychol 80:381-396.

Pessoa L, Kastner S, Ungerleider LG (2002a) Attentional control of the processing of neutral and emotional stimuli. Brain Res Cognit Brain Res 15:31-45.

Pessoa L, McKenna M, Gutierrez E, Ungerleider LG (2002b) Neural processing of emotional faces requires attention. Proc Natl Acad Sci USA 99:11458-11463.
Phelps EA, O'Connor KJ, Gatenby JC, Gore JC, Grillon C, Davis M (2001) Activation of the left amygdala to a cognitive representation of fear. Nat Neurosci 4:437-441.

Phillips ML, Young AW, Senior C, Brammer M, Andrew C, Calder AJ, Bullmore ET, Perrett DI, Rowland D, Williams SC, Gray JA, David AS (1997) A specific neural substrate for perceiving facial expressions of disgust. Nature 389:495-498.

Phillips ML, Young AW, Scott SK, Calder AJ, Andrew C, Giampietro V, Williams SC, Bullmore ET, Brammer M, Gray JA (1998) Neural responses to facial and vocal expressions of fear and disgust. Proc R Soc Lond B Biol Sci 265:1809-1817.

Rozin P, Fallon AE (1987) A perspective on disgust. Psychol Rev 94:23-41.

Sawchuk CN, Meunier SA, Lohr JM, Westendorf DH (2002) Fear, disgust, and information processing in specific phobia: the application of signal detection theory. J Anxiety Disord 16:495-510.

Sheline YI, Barch DM, Donnelly JM, Ollinger JM, Snyder AZ, Mintun MA (2001) Increased amygdala response to masked emotional faces in depressed subjects resolves with antidepressant treatment: an fMRI study. Biol Psychiatry 50:651-658.

Shi C, Davis M (1999) Pain pathways involved in fear conditioning measured with fear-potentiated startle: lesion studies. J Neurosci 19:420-430.

Sprengelmeyer R, Young AW, Calder AJ, Karnat A, Lange H, Homberg V, Perrett DI, Rowland D (1996) Loss of disgust: perception of faces and emotions in Huntington's disease. Brain 119:1647-1665.

Thompson RF (1962) The role of the cerebral cortex in stimulus generalization. J Comp Physiol Psychol 55:279-287.

Vuilleumier P, Armony JL, Driver J, Dolan RJ (2001) Effects of attention and emotion on face processing in the human brain: an event-related fMRI study. Neuron 30:829-841.

Vuilleumier P, Armony J, Clarke K, Husain M, Driver J, Dolan R (2002) Neural response to emotional faces with and without awareness: eventrelated fMRI in a parietal patient with visual extinction and spatial neglect. Neuropsychologia 40:2156.

Ware J, Jain K, Burgess I, Davey GC (1994) Disease-avoidance model: factor analysis of common animal fears. Behav Res Ther 32:57-63.

Whalen PJ (1998) Fear, vigilance, and ambiguity: initial neuroimaging studies of the human amygdala. Curr Direct Psychol Sci 7:177-188.

Whalen PJ, Rauch SL, Etcoff NL, McInerney SC, Lee MB, Jenike MA (1998) Masked presentations of emotional facial expressions modulate amygdala activity without explicit knowledge. J Neurosci 18:411-418.

Williams JMG, Mathews A, MacLeod C (1996) The emotional Stroop task and psychopathology. Psychol Bull 120:3-24.

Wojciulik E, Kanwisher N, Driver J (1998) Covert visual attention modulates face-specific activity in the human fusiform gyrus: fMRI study. J Neurophysiol 79:1574-1578.

Yeshurun Y, Carrasco M (1998) Attention improves or impairs visual performance by enhancing spatial resolution. Nature 396:72-75. 